\title{
The Effect of Proprioceptive Neuromuscular Facilitation on Joint Position Sense: A Systematic Review
}

\author{
Hiroshi Takasaki, Yu Okubo, and Shun Okuyama
}

\begin{abstract}
Context: Accurate joint position sense (JPS) is necessary for effective motor learning and high performance in activities that require fine motor control. Proprioceptive neuromuscular facilitation (PNF) can be a promising intervention. Objective: To examine existing peer-reviewed original studies that have investigated the effect of PNF techniques on the JPS in terms of the methodological quality, PNF techniques, outcomes, and participant characteristics. Evidence Acquisition: A systematic literature search was performed using PubMed, EMBASE, MEDLINE, CINAHL, SocINDEX, Scopus, and Cochrane Library from inception to January 2018. The following inclusion criteria were used: (1) assessment of the JPS; (2) peer-reviewed original studies with a randomized controlled trial or quasi-randomized controlled trial design; (3) participants with musculoskeletal disorders or healthy individuals (ie, neither animal studies nor those involving neurological problems); and (4) no cointervention with PNF, except for warm-up procedures. The methodological quality was assessed using PEDro scale and 5 additional criteria. Effect size $\left(\eta^{2}\right)$ was calculated where a positive value indicated an increased JPS after PNF as compared with other approaches including the wait-and-see method. Evidence Synthesis: Nine studies were examined for their methodological quality, and only one study scored $>6$ on the PEDro scale. Positive and large effect size $\left(\eta^{2}>.14\right)$ was detected in 2 studies where JPS of the knee with contract-relax and replication techniques was assessed in healthy individuals. However, the methodological quality of these studies was poor (PEDro scores of 3 and $\leq 5$ in the total quality score out of 16 , respectively). Conclusions: The current study did not find multiple studies with high methodological quality and similar PNF techniques, outcomes, and characteristics of participants. More high-quality studies are required to achieve a comprehensive understanding of the effect of PNF on the JPS.
\end{abstract}

Keywords: proprioception, proprioceptive neuromuscular facilitation (PNF) stretching, physical therapy techniques

Proprioceptive deficits including impaired joint position sense (JPS) are considered to be contributing to impaired neuromuscular control, resulting in functional instability and repetitive injury. ${ }^{1}$ Furthermore, impaired JPS is associated with impaired muscle performance, ${ }^{2}$ and this combination is associated with functional deficits in people with musculoskeletal disorders. ${ }^{3}$ Therefore, it is important to identify effective interventions to enhance the JPS for preventing injuries and restoring functions in sports rehabilitation and musculoskeletal physiotherapy. Furthermore, sports performance can be associated with the magnitude of the JPS. ${ }^{4}$ Therefore, interventions to enhance the JPS would be concerns in the area of athletic training and sports science. Proprioceptive neuromuscular facilitation (PNF) can be a promising intervention, and several studies have investigated this subject in the past 15 years. ${ }^{5-7}$

$\mathrm{PNF}$ is an applied technique designed to promote the response of the neuromuscular mechanisms, such as mobility, muscular strength and endurance, joint stability, balance, and neuromuscular control, ${ }^{8}$ by stimulating the proprioceptors within the skin, joints, muscles, and tendons. There are several PNF techniques commonly mentioned in the literature, including the contract-relax method and the contract-relax-antagonist-contract method to improve mobility, ${ }^{9}$ as well as the repeated contraction method and the replication method to facilitate neuromuscular outputs. Several systematic reviews have discussed the effect of the contract-relax and

Takasaki and Okuyama are with the Department of Physical Therapy, Saitama Prefectural University, Koshigaya, Japan. Okubo is with the Department of Physical Therapy, School of Physical Therapy, Faculty of Health and Medical Care, Saitama Medical University, Iruma, Japan. Takasaki (physical.therapy.takasaki@gmail. com) is corresponding author. contract-relax-antagonist-contract methods on the performance in power dominant tasks and endurance dominant tasks. ${ }^{10,11}$ Generally, the 2 methods tend to reduce performance in the power dominant tasks and endurance dominant tasks. However, to our knowledge, thus far, no systematic review has investigated the effect of PNF on the performance of JPS. Studies found that PNF enhanced activities of the superior parietal cortex ${ }^{12}$ and cerebellum, ${ }^{13}$ both of which contribute to the JPS.

It may be possible that PNF contributes to enhancement of the JPS, but the effect of PNF on the JPS is hypothesized to differ with different techniques, body regions, and physical problems. A meta-analysis involving several high-quality studies that involve similar techniques, outcome measures, and patient characteristics is required to comprehensively understand the effect of an intervention; however, it is unclear as to how many such studies are available. The present study aimed to examine existing peerreviewed original studies that have investigated the effect of PNF techniques on the JPS in terms of the methodological quality, PNF techniques, outcomes, and participant characteristics. The findings of this study provide direction for future researches aimed at achieving a comprehensive understanding of the effect of PNF techniques on the JPS.

\section{Methods}

\section{Design}

This systematic review was undertaken in accordance with the Preferred Reporting Items for Systematic Reviews and Meta-Analyses guidelines ${ }^{14}$ and updated method guidelines for Cochrane Musculoskeletal Group systematic reviews and meta- 
analyses. ${ }^{15}$ This study was registered in the international prospective register of systematic reviews (CRD42016041823).

\section{Identification and Selection of Studies}

A systematic search was performed using the refined key search terms (Appendix 1) on PubMed, EMBASE, MEDLINE, CINAHL, SocINDEX, Scopus, and the Cochrane Library from inception to January 2018. Cross-referencing was undertaken using the relevant reviews.

The following inclusion criteria were used: (1) assessment of the JPS; (2) peer-reviewed original studies with a randomized controlled trial or quasi-randomized controlled trial design; (3) participants with musculoskeletal disorders or healthy individuals (ie, neither animal studies nor those involving neurological problems); and (4) no cointervention with PNF, except for warm-up procedures. There was no language limitation. One author (H.T.) undertook database search. Screening of the title, abstract, and full text was performed by 2 authors (S.O. and Y.O.) independently. Full-text inspection was undertaken for the studies that either of the authors retained for full-text inspection during the screening process. There was no disagreement regarding the inclusion of the studies as per the inclusion criteria.

\section{Assessment of Methodological Quality}

We used the PEDro scale. ${ }^{16-18}$ In the PEDro scale, a high-quality study is defined as a study with a PEDro score $\geq 6 .{ }^{17}$ Reducing the cutoff score from 6 to 5 did not affect the overall outcome, and a cutoff score of 5 was used in some reviews. ${ }^{19-21}$ Therefore, we investigated if the number of high-quality studies changed using the 2 thresholds.

To further understand the methodological quality, the following 5 points were also assessed (Appendix 2): (1) approval from the ethics committee and informed consent (criterion A), (2) trial registration (criterion B), (3) inclusion of an adequate sample size (criterion C), (4) reliability of the outcome measures (criterion D), and (5) appropriate statistical assessment with effect size calculation (repeated-measures analysis of variance with effect size calculation for parametric analysis and Mann-Whitney $U$ test using predifference to postdifference with effect size calculation; criterion E). These criteria have been used in other critical appraisal tools and CONSORT statement. ${ }^{22,23}$

The International Committee of Medical Journal Editors requested that all clinical trials be registered in the database before patient recruitment in 2005 . Therefore, we evaluated if trial registration was undertaken for studies published after 2005.

The 2 authors (S.O. and Y.O.) assessed the methodological quality, and any disagreement between them was resolved by another author (H.T.). Agreement regarding the PEDro scale and the additional criteria between the 2 authors (S.O. and Y.O.) was examined with Cohen kappa and percentage agreement, where the following interpretations of the kappa value were used: $<.4=$ poor agreement, .41 to $.60=$ moderate agreement, .61 to $.80=$ good agreement, and .81 to $1.0=$ very good agreement. ${ }^{24}$ All the authors were certified in the PEDro scale training program.

\section{Data Analyses}

Data were extracted by the 2 authors (S.O. and H.T.) independently. Any disagreement was resolved by another author (Y.O.). Effect size $\left(\eta^{2}\right)$ was calculated, where $\eta^{2}$ values of $<.003, .01$ to .039 , .06 to .11 and >.14 represent no effect, small effect, intermediate effect, and large effect size, respectively. ${ }^{25}$ A positive value in the effect size indicated increased JPS after PNF compared with that with other approaches (control groups), including the wait-and-see method. Data of the control group were extracted from the wait-and-see method when it was reported; otherwise, data of the control groups were extracted from other approaches compared with the PNF group. A negative effect size value indicated decreased JPS after PNF compared with that with the control groups. Effect size $\left(\eta^{2}\right)$ for the interaction effect and intervention effect was calculated. When the effect size $\left(\eta^{2}\right)$ for the interaction effect and/or the intervention effect was not reported, the $\eta^{2}$ value between the PNF group and the control group was calculated using the within-group change scores. Furthermore, when the within-group change scores were not reported and when the baseline scores were assumed to be comparable, the $\eta^{2}$ value between the PNF group and the control group was calculated using the postintervention scores. ${ }^{19}$ The corresponding author of the literature was contacted twice via e-mail within a 1-month interval in case of insufficient information for data extraction.

\section{Results}

\section{Study Selection}

Figure 1 presents the flow of study selection. Nine studies were assessed for the methodological quality. Lazarou et al ${ }^{5}$ provided us with unpublished data of effect sizes.

\section{Characteristics of the Studies}

Table 1 summarizes the 9 studies. Two studies were published in Japanese, while the others were in English.

Table 2 presents the PEDro scores and additional criteria A to E. For the PEDro scale, the reviewers scored 99 items and agreed on 96 items (97.0\% agreement). The kappa value with $95 \%$ confidence intervals was .94 (.87-1.00) $(P<.001)$, and the overall interreviewer agreement of the methodological quality was good. With respect to the additional criteria $\mathrm{A}$ to $\mathrm{E}$ scale, the reviewers scored 45 items and agreed on all the items (100\% agreement). The kappa value with $95 \%$ confidence intervals was 1.0 (1.0) $(P<.001)$, and the overall interreviewer agreement of the methodological quality was good. More than $80 \%$ of studies failed to fulfill criterion 3 (concealed allocation), criterion 5 (subject blinding), criterion 6 (therapist blinding), criterion 9 (intention-to-treat analysis), criterion B (trial registration), criterion C (inclusion of an adequate sample size), and criterion $\mathrm{E}$ (appropriate statistical assessment with effect size calculation). Regardless of the PEDro cutoff score (5 or 6), 6 out of 9 studies were not considered to be of high quality. Furthermore, none of the studies satisfied all the additional criteria (criteria A-E). All the studies except the one by Padua et $\mathrm{al}^{7}$ were published after 2005; however, only one study ${ }^{5}$ registered the trial.

\section{Discussion}

The current study systematically reviewed the studies that investigated the effect of PNF techniques on the JPS in terms of methodological quality, PNF techniques, outcomes, and participant characteristics. This current review provides us suggestions for enhancing the methodological quality by understanding the common errors in existing studies and gaps in the literature. 


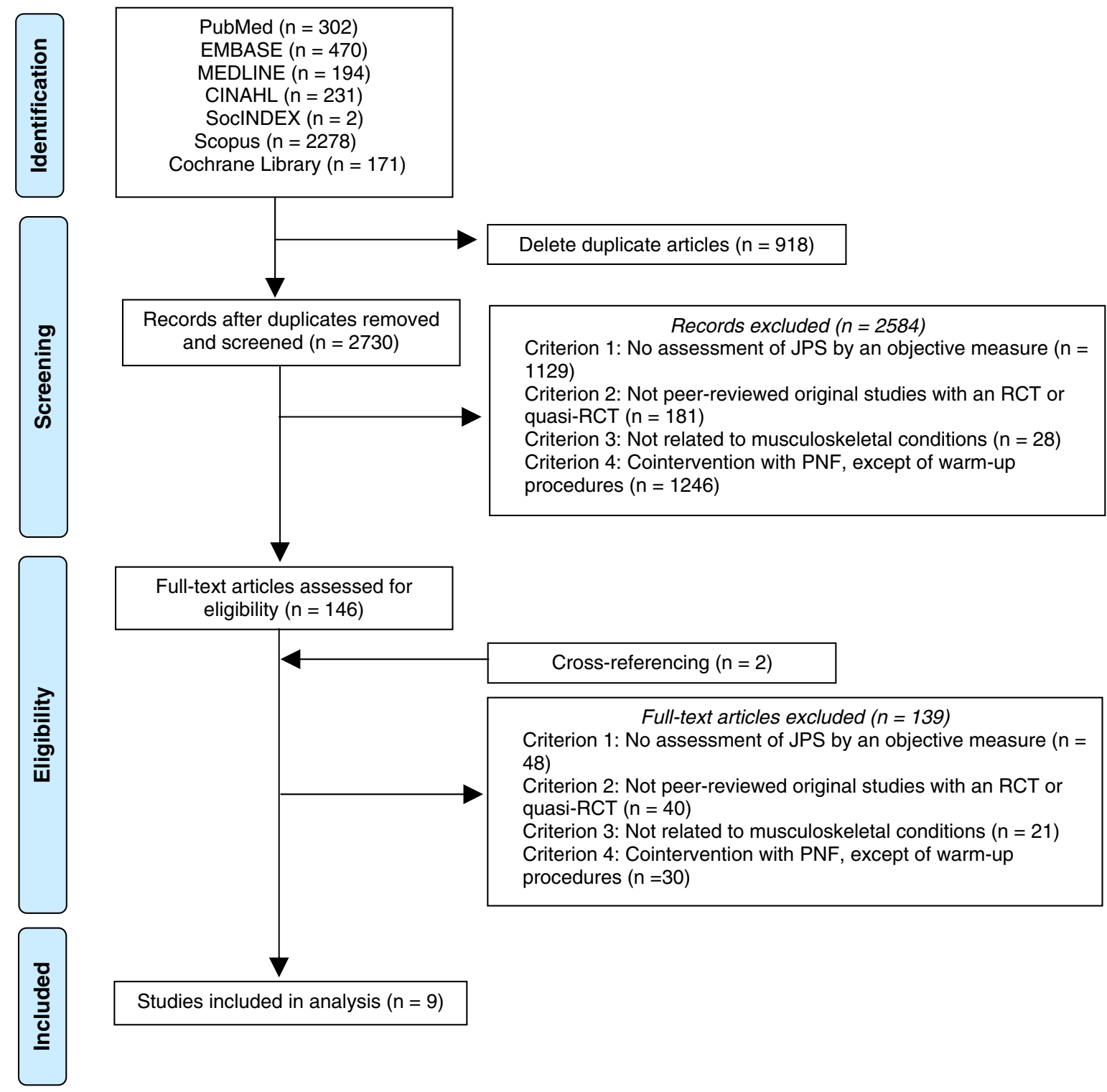

Figure 1 - Flow of the study selection. JPS indicates joint position sense; RCT, randomized controlled trial.

Regardless of the PEDro cutoff score, 6 out of 9 studies were not considered to be of high quality. Furthermore, none of the studies satisfied all the additional criteria. Even in the study by Lazarou et al, ${ }^{5}$ wherein the methodological quality was considered high as per the PEDro score, additional criteria C (inclusion of an adequate sample size) and D (reliability of the outcome measures) were not satisfied. These faults in the additional criteria can influence conclusions of the study ${ }^{5}$; thus, the results should be cautiously interpreted. No study satisfied criterion C (inclusion of an adequate sample size) and criterion $\mathrm{E}$ (appropriate statistical assessment with effect size calculation). Underpowered results and inappropriate statistical assessment or the lack of effect size calculation influenced the study conclusions. Furthermore, it was found that most studies in the current review did not register the trial and did not follow global publication standards. Therefore, we recommend that we should assume the lack of convincing information about the effect of PNF on the JPS. In order to fully understand the effect of PNF on the JPS, further well-designed studies are required.

\section{Considerations for Future Studies}

We calculated the effect sizes for the effect of PNF on the JPS in each study. A large effect size was observed in 2 studies ${ }^{6,31}$ although the methodological quality was poor in each study. These studies suggest promising conditions that can be used in future trials. First, regarding a target joint, the 2 studies with a large effect size $^{6,31}$ used the knee. Thus, the knee can be a promising target in future trials.

Regarding the PNF technique, Cho et $\mathrm{al}^{6}$ used contract-relax and Ito et $\mathrm{al}^{31}$ used replication. Only the postintervention scores were available from the Cho et al's study6; thus, effect size was calculated using the postintervention scores. In the study by Ito et al, ${ }^{31}$ only within-group changes in the scores were available; 


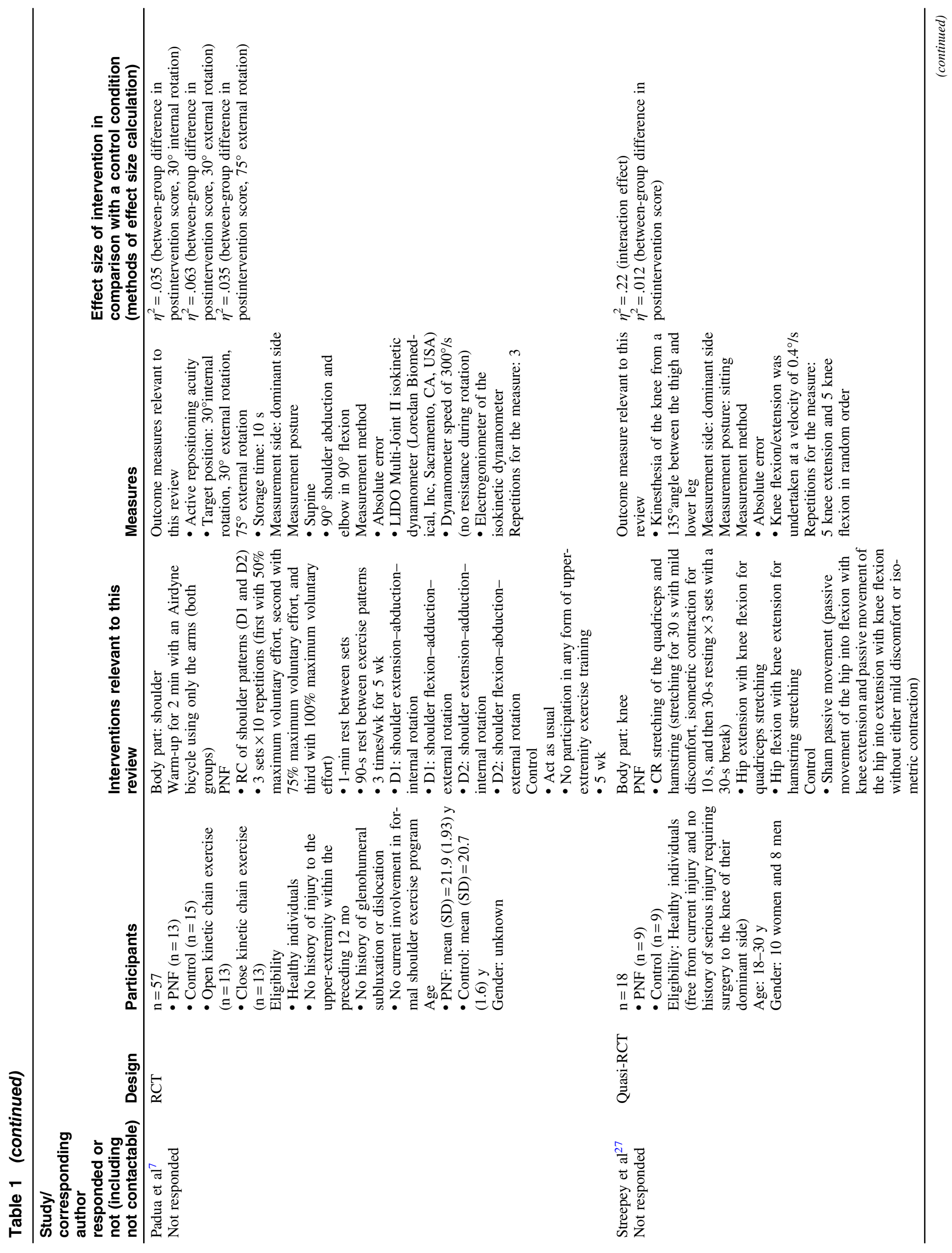




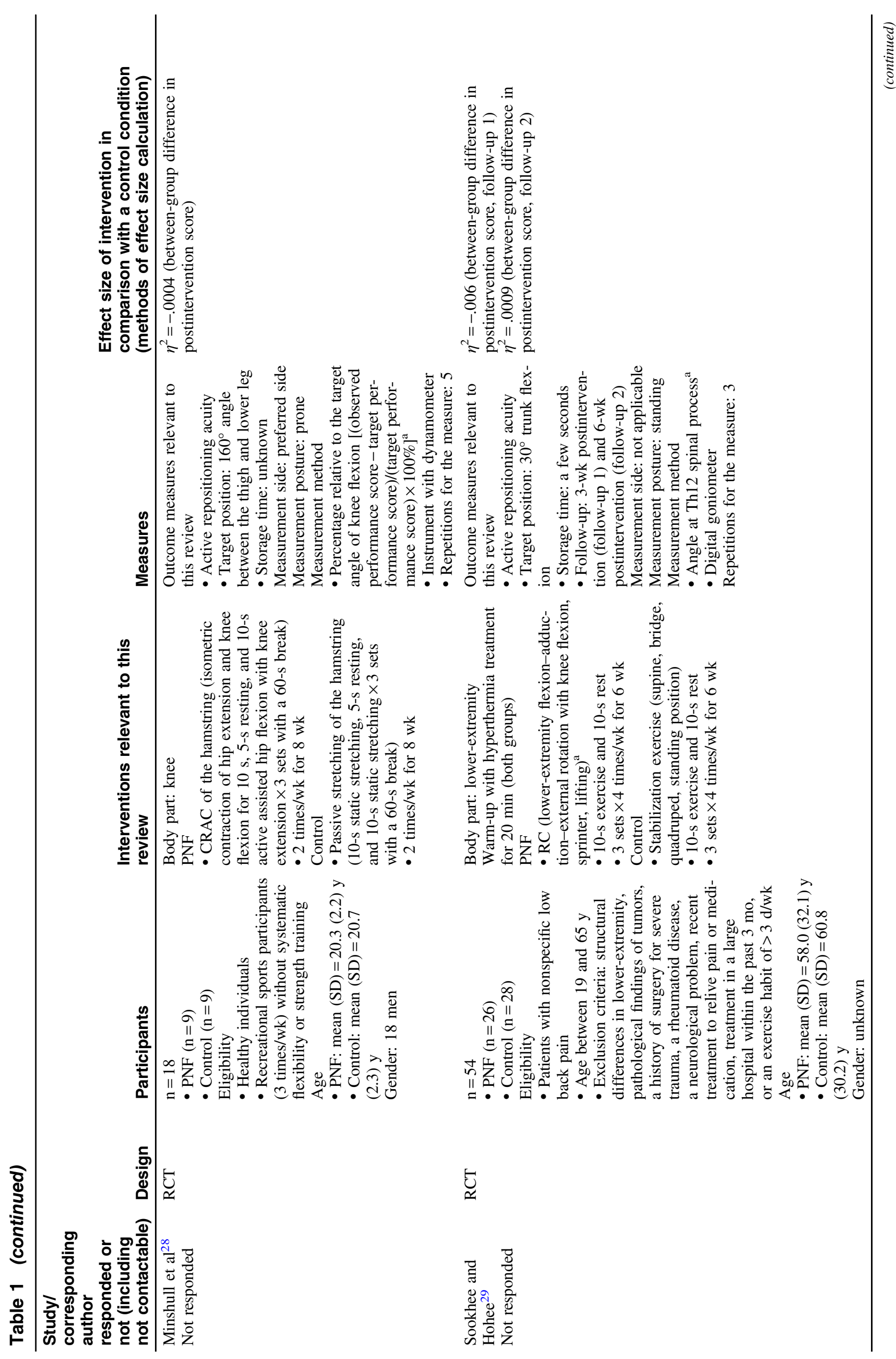




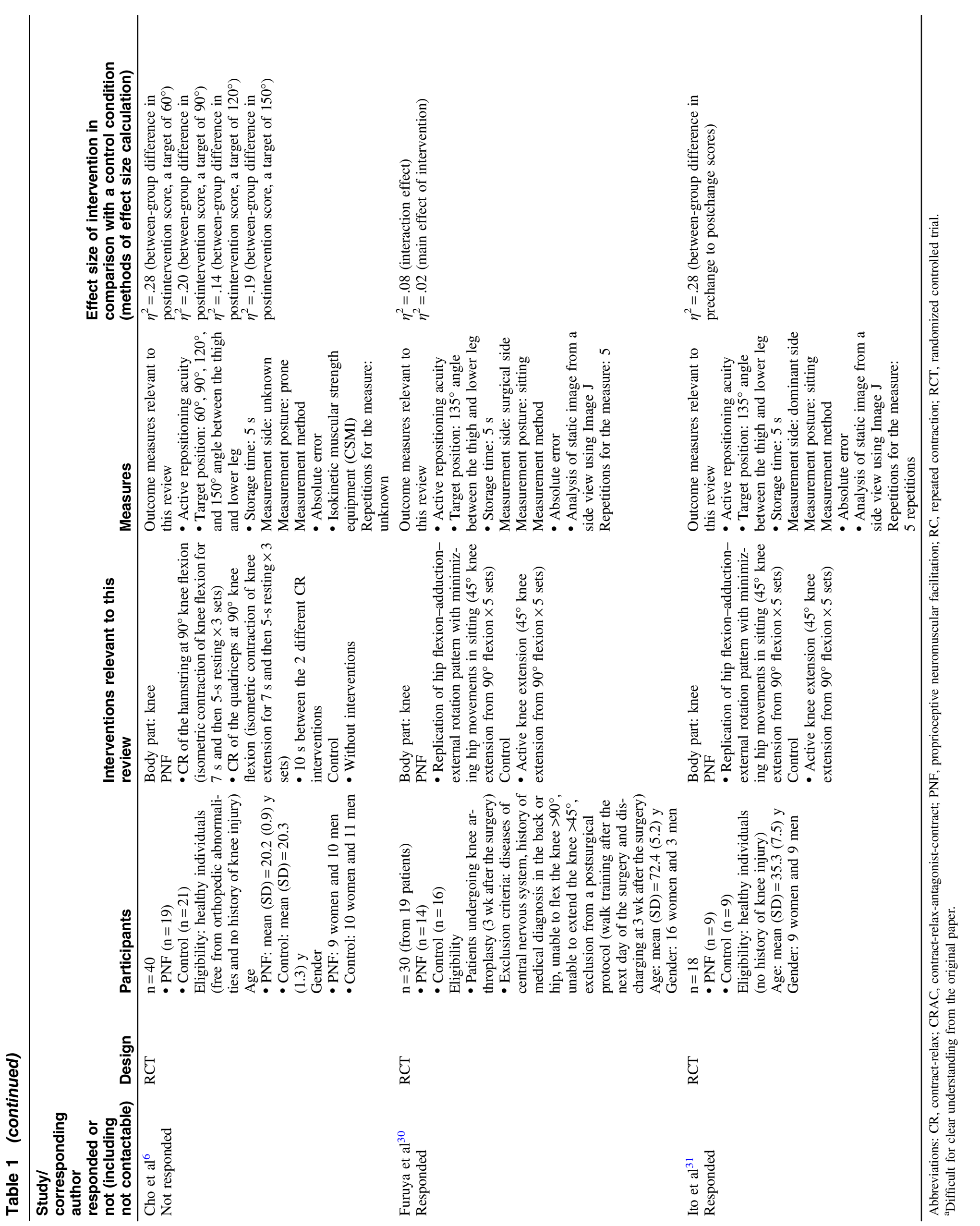


Table 2 Methodological Quality

\begin{tabular}{|c|c|c|c|c|c|c|c|c|c|c|c|c|c|c|c|c|c|c|}
\hline \multirow[b]{2}{*}{ Study } & \multicolumn{11}{|c|}{ PEDro criteria } & \multicolumn{5}{|c|}{ Additional criteria } & \multirow[b]{2}{*}{$\begin{array}{l}\text { PEDro total from } \\
\text { criteria } 2 \text { to } 11(/ 10)\end{array}$} & \multirow[b]{2}{*}{$\begin{array}{l}\text { Total } \\
(/ 16)\end{array}$} \\
\hline & 1 & 2 & 3 & 4 & 5 & 6 & 7 & 8 & 9 & 10 & 11 & A & B & C & D & $\mathbf{E}$ & & \\
\hline Lazarou et $\mathrm{al}^{5}$ & 1 & 1 & 1 & 1 & 1 & 1 & 1 & 1 & 0 & 1 & 1 & 1 & 1 & 0 & 0 & 1 & 9 & 13 \\
\hline Bjorklund et $\mathrm{al}^{26}$ & 1 & 1 & 0 & 1 & 0 & 0 & 0 & 1 & 0 & 1 & 1 & 1 & 0 & 0 & 1 & 0 & 5 & 8 \\
\hline Padua et $\mathrm{al}^{7}$ & 0 & 1 & 0 & 1 & 0 & 0 & 0 & 1 & 0 & 1 & 1 & 1 & 0 & 0 & 1 & 0 & 5 & 7 \\
\hline Streepey et $\mathrm{al}^{27}$ & 0 & 0 & 0 & 1 & 0 & 0 & 0 & 1 & 0 & 1 & 1 & 1 & 0 & 0 & 1 & 0 & 4 & 6 \\
\hline Minshull et $\mathrm{al}^{28}$ & 0 & 1 & 0 & 1 & 0 & 0 & 0 & 0 & 0 & 1 & 1 & 1 & 0 & 0 & 0 & 0 & 4 & 5 \\
\hline Sookhee and Hohee 29 & 1 & 1 & 0 & 1 & 0 & 0 & 0 & 0 & 0 & 1 & 1 & 0 & 0 & 0 & 0 & 0 & 4 & 5 \\
\hline Cho et $\mathrm{al}^{6}$ & 1 & 1 & 0 & 0 & 0 & 0 & 0 & 1 & 0 & 1 & 0 & 1 & 0 & 0 & 0 & 0 & 3 & 5 \\
\hline Furuya et $\mathrm{al}^{30}$ & 0 & 1 & 0 & 0 & 0 & 0 & 1 & 0 & 0 & 1 & 1 & 0 & 0 & 0 & 0 & 0 & 4 & 4 \\
\hline Ito et $\mathrm{al}^{31}$ & 0 & 1 & 0 & 1 & 0 & 0 & 0 & 0 & 0 & 1 & 0 & 0 & 0 & 0 & 0 & 0 & 3 & 3 \\
\hline
\end{tabular}

Note: A greater total score generally indicates a better methodological quality $(0=$ not satisfied and $1=$ satisfied $)$.

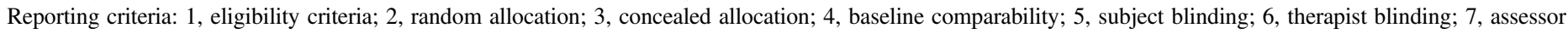

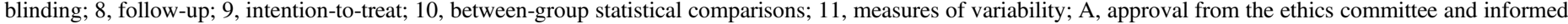

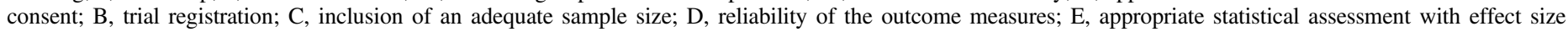
calculation.

thus, the effect size was calculated based on the within-group change scores. Statistically, an effect size calculated using the within-group change scores is considered more accurate than that calculated using the postintervention scores. Therefore, a promising intervention in a future trial may be a replication, and this would not be surprising considering findings in previous studies that investigated brain area facilitated by PNF. ${ }^{12,13}$ The replication includes rotational elements of joint movements, and all previous studies that demonstrated enhanced activities of the superior parietal cortex and cerebellum ${ }^{12,13}$ used PNF techniques with rotational elements. Therefore, rotational elements of movements may be a key factor for PNF techniques to enhance the JPS, and future studies are required.

Regarding participants, the 2 studies with a large effect size $e^{6,31}$ recruited healthy individuals. When we explored the possibilities of enhancing the performance in activities that require fine motor control with PNF, a study involving healthy individuals may be considered. By contrast, although Furuya et $\mathrm{al}^{30}$ and Ito et $\mathrm{al}^{31}$ used replication targeting the knee, Furuya et $\mathrm{al}^{30}$ did not detect an effect size as large as that in Ito et al's study. ${ }^{31}$ This difference may have been associated with the difference in the recruited participants because Furuya et $\mathrm{al}^{30}$ recruited patients undergoing knee arthroplasty, while Ito et $\mathrm{al}^{31}$ recruited healthy individuals. When we explored effective interventions to improve impaired JPS, a trial with participants with more preserved mechanoreceptors than that in patients undergoing knee arthroplasty would be demanded. It has been known that patients with anterior cruciate ligament injury have impaired JPS, ${ }^{32-35}$ and thus, it may be important to undertake a future trial including patients with anterior cruciate ligament injuries to investigate effect of replication technique on the JPS.

\section{Study Limitations}

The current review has 2 limitations. First, the ideal effect size calculation should have been performed with the interaction effect and intervention effect. However, such a statistical analysis was reported by Furuya et $\mathrm{al}^{30}$ only. It was impossible to obtain additional data for the statistical analyses, and effect size calculations were undertaken using the within-group change scores or the postinter-

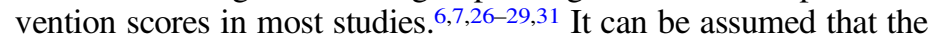

calculated effect sizes in the current study can be different from the genuine effect sizes. The use of the effect sizes is recommended only for future research designs. Second, there were 2 studies with unclear information regarding the interventions and/or outcomes, ${ }^{28,29}$ where it was impossible to clarify the information from the corresponding authors. We could not reperform these studies, and these results should be cautiously interpreted.

\section{Conclusions}

We found that there were no multiple studies with high methodological quality and similar PNF techniques, outcomes, and participant characteristics. More high-quality studies are required to achieve a comprehensive understanding regarding the effect of PNF on the JPS.

\section{References}

1. Lephart SM, Pincivero DM, Giraldo JL, Fu FH. The role of proprioception in the management and rehabilitation of athletic injuries. Am J Sports Med. 1997;25:130-137. PubMed ID: 9006708 doi:10.1177/ 036354659702500126

2. Mota N, Ribeiro F. Association between shoulder proprioception and muscle strength in water polo players. Isokinet Exerc Sci. 2012;20: 17-21. doi:10.3233/IES-2011-0435

3. van der Esch M, Steultjens M, Harlaar J, Knol D, Lems W, Dekker J. Joint proprioception, muscle strength, and functional ability in patients with osteoarthritis of the knee. Arthritis Rheum. 2007;57: 787-793. PubMed ID: 17530678 doi:10.1002/art.22779

4. Kaya D, Callaghan MJ, Donmez G, Doral MN. Shoulder joint position sense is negatively correlated with free-throw percentage in professional basketball players. Isokinet Exerc Sci. 2012;20:189196. doi:10.3233/IES-2012-0458

5. Lazarou L, Kofotolis N, Malliou P, Kellis E. Effects of two proprioceptive training programs on joint position sense, strength, activation and recurrent injuries after ankle sprains. Isokinet Exerc Sci. 2017; 25:289-300. doi:10.3233/IES-171146

6. Cho SI, Lee DY, Hong JH, Yu JH, Kim JS. Effect of hold and relax technique on knee joint position awareness in normal adults. Indian $J$ Sci Technol. 2015;8(19). 
7. Padua DA, Guskiewicz KM, Prentice WE, Schneider RE, Shields EW. The effect of select shoulder exercises on strength, active angle reproduction, single-arm balance, and functional performance. J Sport Rehabil. 2004;13:75-95. doi:10.1123/jsr.13.1.75

8. Sharman MJ, Cresswell AG, Riek S. Proprioceptive neuromuscular facilitation stretching: mechanisms and clinical implications. Sports Med. 2006;36:929-939. PubMed ID: 17052131 doi:10.2165/00007256200636110-00002

9. Hindle KB, Whitcomb TJ, Briggs WO, Hong J. Proprioceptive neuromuscular facilitation (PNF): its mechanisms and effects on range of motion and muscular function. J Hum Kinet. 2012;31: 105-113. PubMed ID: 23487249 doi:10.2478/v10078-012-0011-y

10. Peck E, Chomko G, Gaz DV, Farrell AM. The effects of stretching on performance. Curr Sports Med Rep. 2014;13:179-185. PubMed ID: 24819010 doi:10.1249/JSR.0000000000000052

11. Behm DG, Blazevich AJ, Kay AD, McHugh M. Acute effects of muscle stretching on physical performance, range of motion, and injury incidence in healthy active individuals: a systematic review. Appl Physiol Nutr Metab. 2016;41:1-11. PubMed ID: 26642915 doi:10.1139/apnm-2015-0235

12. Lial L, Moreira R, Correia L, et al. Proprioceptive neuromuscular facilitation increases alpha absolute power in the dorsolateral prefrontal cortex and superior parietal cortex. Somatosens Mot Res. 2017;34:204-212. PubMed ID: 29096587 doi:10.1080/08990220. 2017.1392298

13. Shuratani T, Nitta O, Matsuda M, Tada Y, Senoo A, Yanagisawa K. The effects of a static contraction of pelvic anterior elevation on the brain activities induced by a fMRI in the normal volunteers. $J$ Jpn Acad Health Sci. 2011;14:205-212.

14. Moher D, Liberati A, Tetzlaff J, Altman DG, PRISMA Group. Preferred reporting items for systematic reviews and meta-analyses: the PRISMA statement. Int J Surg. 2010;8:336-341. PubMed ID: 20171303 doi:10.1016/j.ijsu.2010.02.007

15. Ghogomu EA, Maxwell LJ, Buchbinder R, et al. Updated method guidelines for Cochrane Musculoskeletal Group systematic reviews and metaanalyses. J Rheumatol. 2014;41(2):194-205. PubMed ID: 24293581 doi:10.3899/jrheum.121306

16. de Morton NA. The PEDro scale is a valid measure of the methodological quality of clinical trials: a demographic study. Aust J Physiother. 2009;55:129-133. PubMed ID: 19463084 doi:10.1016/ S0004-9514(09)70043-1

17. Maher CG, Sherrington C, Herbert RD, Moseley AM, Elkins M. Reliability of the PEDro scale for rating quality of randomized controlled trials. Phys Ther. 2003;83:713-721. PubMed ID: 12882612

18. Sherrington C, Herbert RD, Maher CG, Moseley AM. PEDro. A database of randomized trials and systematic reviews in physiotherapy. Man Ther. 2000;5:223-226. PubMed ID: 11052901 doi:10. 1054/math.2000.0372

19. Takasaki H, May S. Mechanical diagnosis and therapy has similar effects on pain and disability as 'wait and see' and other approaches in people with neck pain: a systematic review. J Physiother. 2014;60: 78-84. PubMed ID: 24952834 doi:10.1016/j.jphys.2014.05.006

20. Boyles R, Toy P, Mellon J Jr, Hayes M, Hammer B. Effectiveness of manual physical therapy in the treatment of cervical radiculopathy: a systematic review. J Man Manip Ther. 2011;19:135-142. PubMed ID: 22851876 doi:10.1179/2042618611Y.0000000011

21. Coppola SM, Collins SM. Is physical therapy more beneficial than unsupervised home exercise in treatment of post surgical knee disorders? A systematic review. Knee. 2009;16:171-175. PubMed ID: 18851916 doi:10.1016/j.knee.2008.09.001

22. Takasaki H, Lim ECW, Soon B. The effect of shoulder muscle fatigue on active repositioning acuity and scapulothoracic resting alignment: a systematic review with meta-analysis. Phys Ther Sport. 2016;20: 61-78. PubMed ID: 27080109 doi:10.1016/j.ptsp.2016.01.001

23. Takasaki H, Miki T. The impact of continuous use of lumbosacral orthoses on trunk motor performance: a systematic review with metaanalysis. Spine J. 2017;17:889-900. PubMed ID: 28323240 doi:10. 1016/j.spinee.2017.03.003

24. Altman DG. Practical Statistics for Medical Research. London, UK: Chapman \& Hall; 1991.

25. Cohen J. Statistical Power Analysis for the Behavioural Sciences. 2nd ed. Hillsdale, NJ: Lawrence Erlbaum Associates, Inc.; 1988.

26. Bjorklund M, Djupsjobacka M, Crenshaw AG. Acute muscle stretching and shoulder position sense. J Athl Train. 2006;41:270-274. PubMed ID: 17043694

27. Streepey JW, Mock MJ, Riskowski JL, Vanwye WR, Vitvitskiy BM, Mikesky AE. Effects of quadriceps and hamstrings proprioceptive neuromuscular facilitation stretching on knee movement sensation. J Strength Cond Res. 2010;24:1037-1042. PubMed ID: 20300021 doi:10.1519/JSC.0b013e3181d09e87

28. Minshull C, Eston R, Bailey A, Rees D, Gleeson N. The differential effects of PNF versus passive stretch conditioning on neuromuscular performance. Eur J Sport Sci. 2014;14:233-241. PubMed ID: 23688197 doi:10.1080/17461391.2013.799716

29. Sookhee B, Hohee S. The effects of proprioceptive neuromuscular facilitation and stabilizing exercise on trunk repositioning errors. J Phys Ther Sci. 2012;24:1017-1020. doi:10.1589/jpts.24.1017

30. Furuya H, Ito T, Sumiya K, Tomoshige S, Tanaka T. The effect of $\mathrm{PNF}$ techniques with replication for the joint position sense in patients undergoing knee arthroplasty. PNF Res. 2009;10:28-32.

31. Ito T, Saito T, Sato H, Shinoda M. The influence of replication on knee joint kinesthetic sense. PNF Res. 2007;7:39-43.

32. Relph N, Herrington L. Knee joint position sense ability in elite athletes who have returned to international level play following ACL reconstruction: a cross-sectional study. Knee. 2016;23:1029-1034. PubMed ID: 27712856 doi:10.1016/j.knee.2016.09.005

33. Friden T, Roberts D, Ageberg E, Walden M, Zatterstrom R. Review of knee proprioception and the relation to extremity function after an anterior cruciate ligament rupture. J Orthop Sports Phys Ther. 2001;31:567-576. PubMed ID: 11665744 doi:10.2519/jospt.2001. 31.10 .567

34. Relph N, Herrington L, Tyson S. The effects of ACL injury on knee proprioception: a meta-analysis. Physiotherapy. 2014;100:187-195. PubMed ID: 24690442 doi:10.1016/j.physio.2013.11.002

35. Kim HJ, Lee JH, Lee DH. Proprioception in patients with anterior cruciate ligament tears: a meta-analysis comparing injured and uninjured limbs. Am J Sports Med. 2017;45:2916-2922. PubMed ID: 28060536 doi:10.1177/0363546516682231 


\section{Appendix 1. Search Strategy}

(stretching OR "Proprioceptive Neuromuscular Facilitation") AND (sensorimotor OR proprioception OR “joint position sense")

\section{Appendix 2. Additional Criteria for the Assessment of the Methodological Quality}

[A. Approval from the ethics committee and informed consent]

Have the authors documented the ethical approval for the research and gained informed consent from the participants?

\section{[B. Trial registration]}

Have the authors registered the clinical trial at a clinical trial registry and documented the ID numbers?

\section{[C. Incusion of an adequate sample size]}

Have the authors justified their sample size through a power calculation or post-hoc analysis (and recruited sufficient numbers)?

\section{[D. Reliability of the outcome measures]}

Have the authors documented the evidence of the reliability of the outcome measures relevant to this review?

For studies that have provided references of other works regarding reliability or have demonstrated the accuracy of the outcome measures, this question should be answered with yes.

\section{[E. Appropriate statistical assessment with effect size calculation]}

Did the authors use appropriate statistical analyses for evaluating the results as per their aim, and have they documented the effect size?

When a repeated-measures ANOVA with effect size for parametric analysis and Mann-Whitney $U$ test using pre-post difference with effect size is used, this question should be answered with yes.
Yes

No or Unable to determine

Yes

No or Unable to determine

Yes

No or Unable to determine

Yes

No or Unable to determine

Yes

No or Unable to determine 